\title{
The Features Vector Research on Target Recognition of Airplane
}

\author{
Shuangzi Sun ${ }^{1}$ Lihong Yuan Yong Yang ${ }^{2}$ Xiaochao Chen \\ ${ }^{1}$ Changchun University of Science and Technology, sshz_2000@yahoo.com.cn \\ ${ }^{2}$ Changchun University of Science and Technology, yy@cust.edu.cn
}

\begin{abstract}
The selection of the features vector is crucial, taking direct effect on the accuracy of target recognition.

Considering that the airplane has smooth surface and regular geometric shape, this paper chooses the geometric shape feature to describe the target of airplane. These geometric features vector include the center point, the characteristics of size and shape, the ration of airframe-length to wingspan etc.

Finally, this paper applies Support Vector Machine to validate the effectiveness of geometric features vector. From the result of experiment, it can be concluded that these features vector can satisfy the requirement of target recognition.
\end{abstract}

Keywords: Target Recognition; Features Vector; Geometric Features; Support Vector Machine (SVM)

\section{Introduction}

For the field of image processing, feature extraction and target recognition have a close relation. Feature extraction is based on the research between target and environment characteristic, aim to extract the features expressing the essence attribute of target, these features vector that make it possible to apart the target from environment. The target recognition is based on pattern recognition technology, aim to apart the target form background according to the target features vector that are obtained by feature extraction process, furthermore to ascertain the type of target, its location and another useful information, etc.

The image feature is both the basic content of inner image and the most essential attribute hold by itself and distinguish itself from the other image. The image feature can be natural attribute that human vision can discern, called by nature feature, such as brightness, color, outline, structure etc. The image feature can be man-made attribute, called by man-made feature, normally, the manmade feature is parameter vector that is measured and computed by the researcher, for example, the histogram, the frequency spectrum, the area, and the perimeter etc. In this paper, mainly discuss the manmade feature of the airplane image.

\section{Feature Extraction on Airplane Target}

Normally, the feature of image can be classified for seven groups: (1) the scope feature; (2) statistics feature; (3) transformation feature; (4) boundary feature; (5) topological structure feature; (6) texture feature; (7) geometry feature.

Among of these features, the geometry feature takes an important status for target recognition. To pattern recognition and computer vision, the geometry is the intrinsic characteristic of object. Using the geometry of object can obtain other attributes, such as the borderline of object, the surface normal line etc. At the same

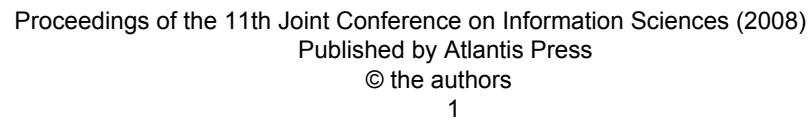


time, the geometry of object can be directly sense by humane visions, so it is easier to extract and process.

The airplane has smooth surface and its geometric shape has certain regulation. Contrast to the outstanding geometric information, texture and the other information are weakness. Thus this paper chooses the geometry feature to describe the target of airplane.

\subsection{The Center Point}

According to practically situation, the target of airplane is impossible to be a single dot in image. Thus, this paper takes the center point of the area to depict the location of the object. The center of the area is defined as $\mathrm{O}$, the center of mass for the image with the same shape and the same quality in unit area. For the binary image $f(x, y)$, if the location of the target's pixel is $\left(x_{i}, y_{i}\right)$, here, $i=0,1, \ldots, N$ $1, \mathrm{j}=0,1, \ldots, \mathrm{M}-1$, then the center of the target can be computed by :

$$
\begin{aligned}
& \bar{x}=\frac{1}{N * M} \sum_{i=0}^{N-1} \sum_{j=0}^{M-1} x_{i} \\
& \bar{y}=\frac{1}{N * M} \sum_{i=0}^{N-1} \sum_{j=0}^{M-1} y_{j}
\end{aligned}
$$

\subsection{The Characteristic of Size}

\subsubsection{The Perimeter and the Area}

Normally, the perimeter of the object can be defined for the boundary length of the target. Thus, for the digital image, the perimeter $\mathrm{L}$ can be computed by the total number of pixels at the boundary of the target. After the border track for the target, it may be hypothesized that the digital curve outline is $\mathrm{P}=\{\mathrm{p} 1, \mathrm{p} 2, \mathrm{p} 3, \mathrm{p} 4, \ldots \mathrm{pn}\}$, here, $\mathrm{n}$ is the number of the pixels on the curve outline, so the perimeter $\mathrm{L}$ is equal to $\mathrm{n}$, namely:

$$
L=n
$$

Subsequently, the parameter of the area is convenient to obtain too. The area of the target is related to the boundary, not to the interior gray level. The easiest method of computation is to count the number of pixels within or on the boundary of the target. For a image with dimension $\mathrm{N}^{*} \mathrm{M}$, if the gray distribution of the target is depicted by $\mathrm{f}(\mathrm{x}, \mathrm{y})$, the area of the target can be computed by:

$$
A_{\text {area }}=\sum_{i=1}^{N} \sum_{j=1}^{M} f\left(x_{i}, y_{j}\right)
$$

To binary value image, supposed that the value of 1 is the object, and 0 for background, the computation of area is to count the number of those pixels that the gray level is 1 .

\subsubsection{Macro Axis and Minor Axis}

When the boundary of the object is known, it is the most simple and effective method to depict the basic shape with the size of the enclosing rectangle. The enclosing rectangle on coordinate orientation can be represented by the span on the horizon and vertical. Aim to the span, just computing the maximum and minimum of the points on the boundary curve. But for the target at random orientation, the horizon and vertical is not crucial. Thus it is necessary to determine the principal axis of the target. By computing the minimum enclosing rectangle, the principal axis can be obtained.

The basic idea of computing the $\operatorname{MER}$ (minimum enclosing rectangle) is rotating the target by the increment of three angles or five angles, within 90 angles. Every rotating, record the maximum and minimum of $\mathrm{x}$ and $\mathrm{y}$ of the enclosing rectangle on this coordinate orientation. Rotating to $\alpha$ angles, the area of the enclosing rectangle is the smallest. Here is MER. And this orientation is the principal axis.

Under this situation, we can compute the macro axis and minor axis. Namely, the macro axis is the width of MER, the minor axis is the height of

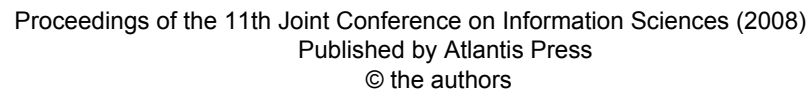


MER. The computation can be depicted by:

LongAxis $=$ MER(width)

ShortAxis $=$ MER(height)

\subsection{The Characteristic of Shape}

\subsubsection{Compactness}

Compactness reflects the proportion of the target to its enclosing rectangle. Namely, compactness is the value that the pixels of the target divide into the pixels of the MER. As above, the $A_{M E R}$ (the area of the MER) is equal to the number of pixels of MER. So, compactness can be depicted by:

$$
J=\frac{A_{\text {area }}}{A_{\text {MER }}}
$$

Here, $A_{\text {area }}$ is the area of the target, $A_{M E R}$ is the area of the MER.

\subsubsection{Complexity}

Complexity is defined as the ration of the number of pixels on the boundary curve to the number of pixels of the wholly target. For the digital image, the number of pixels on the target's boundary is equal to the perimeter $\mathrm{L}$, and the number of the wholly target's pixels is the area $A_{\text {area }}$, so, complexity of the target can be computed by:

$$
F=\frac{L}{A_{\text {area }}}
$$

\subsection{The Ration of Airframe-length to Wingspan}

Aim to obtain the ration of airframe to wingspan, it is necessary to ascertain the direction of the principal axis. The principal axis of airplane is defined as a line that makes the moment of inertia of the region $\mathrm{D}$ the minimum. The moment of inertia of region $\mathrm{D}$ can be computed by:

$$
\begin{aligned}
& I=\iint_{D}\left[(x-\bar{x}) \sin \alpha-(y-\bar{y}]^{2} f(x \cdot y) d x d y\right. \\
& =\sin ^{2} \alpha \iint_{D}(x-\bar{x})^{2} f(x . y) d x d y \\
& +\cos ^{2} \alpha \iint_{D}(y-\bar{y})^{2} f(x, y) d x d y \\
& -2 \sin \alpha \cos \alpha \iint_{D}(x-\bar{x})(y-\bar{y}) f(x, y) d x d y
\end{aligned}
$$

Here, $\alpha$ is inclination of the principal axis through the center $(\bar{x}, \bar{y})$ with the $\mathrm{x}$ axis. As above, the center $(\bar{x}, \bar{y})$ can be computed by Eq.1. And, the integral computation in eq. 6 can be deducted by central moment formula:

$\mu_{p q}=\int_{-\infty}^{+\infty} \int_{-\infty}^{+\infty}(x-\bar{x})^{p}(y-\bar{y})^{q} f(x, y) d x d y$

Therefore, the moment of inertia of region $\mathrm{D}$ may be simplified by:

$$
I=\mu_{20} \sin ^{2} \alpha+\mu_{02} \cos ^{2} \alpha-2 \mu_{11} \sin \alpha \cos \alpha
$$

Want to make the value of I the minimum,

$$
\begin{aligned}
& \frac{d I}{d \alpha}=0, \text { namely, } \\
& \text { then } \sin 2 \alpha-\mu_{02} \sin 2 \alpha-\mu_{11} \cos 2 \alpha=0 \\
& \mu_{20} \sin 2
\end{aligned}
$$

Thereby,

$$
\tan 2 \alpha=\frac{2 \mu_{11}}{\mu_{20}-\mu_{02}}
$$

Then :

$$
\alpha=\frac{1}{2} \tan ^{-1}\left(\frac{2 \mu_{11}}{\mu_{20}-\mu_{02}}\right)
$$

After having the inclination $\alpha$ of the principal axis with the $\mathrm{x}$ axis, the airframe-length is parallel with the $\mathrm{x}$ axis, and the wingspan is parallel with the $y$ axis. The airframe-length $x^{\prime}$ is the projection of the airplane to the $\mathrm{x}$ axis, and the wingspan $y^{\prime}$ is the projection of the airplane to the y axis. Therewith, the ra- 
tion of the airframe-length to wingspan is computed by $T=x^{\prime}, y^{\prime}$.

\section{Experiment}

\subsection{The Result of Features Vector}

Fig. 1 is the top view of six types of airplane. According to equation of features vector above introduced, we can obtain some data of these images. Now, the experiment data are reported as table 1 and table 2 .

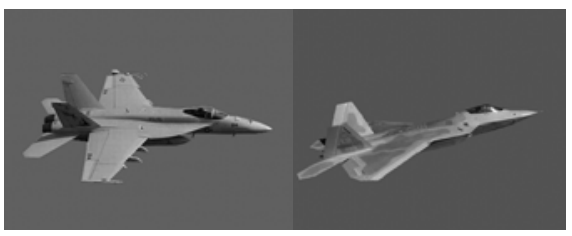

(a) F/A-18

(b) F-22

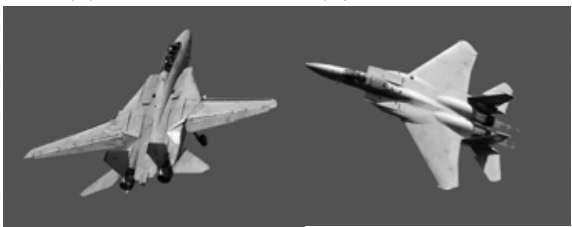

(c) F-14

(d) F-15

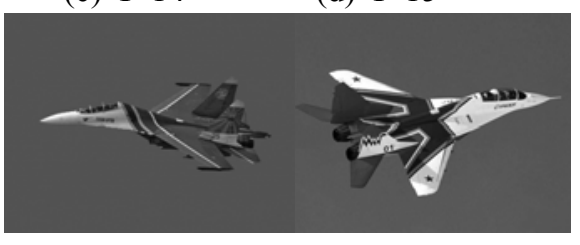

(e) SU-27

(f) Mig-29

Fig. 1 the top vies of six types of airplane

From table 2, SU-27 and F-15 presented negative value. It is normal, because we prescribe that the right direction of the $\mathrm{x}$ axis is positive direction, from images, it is easy to find out that the course of two type of airplane is reverse to the right direction of the $\mathrm{x}$ axis. So the direction of the course is negative. From table 1 and 2, we find out that different type of airplane has different characteristic of shape, especially, the difference of the ration of airframe-length to wingspan is very distinct. Obviously, the ration of airframe-length to wingspan is an important feature vector.

Table1. Experimental data

\begin{tabular}{|c|c|c|c|c|}
\hline \multicolumn{2}{|c|}{3} & F/A-18 & F-22 & F-14 \\
\hline \multirow{2}{*}{$\begin{array}{l}\text { center } \\
\text { point }\end{array}$} & $\mathrm{x}$ & 202.888 & 193.260 & 226.752 \\
\hline & $\mathrm{y}$ & 139.221 & 149.471 & 157.429 \\
\hline \multicolumn{2}{|c|}{ Direction } & 0.023 & 0.207 & 0.474 \\
\hline \multicolumn{2}{|c|}{ Perimeter } & 1080 & 921 & 1248 \\
\hline \multicolumn{2}{|l|}{ Area } & 22243 & 19381 & 29347 \\
\hline \multicolumn{2}{|c|}{ Macro axis } & 393 & 401 & 380 \\
\hline \multicolumn{2}{|c|}{ Minor axis } & 158 & 140 & 226 \\
\hline \multicolumn{2}{|c|}{$\begin{array}{l}\text { Compact- } \\
\text { ness }\end{array}$} & 0.3582 & 0.3756 & 0.2681 \\
\hline \multicolumn{2}{|c|}{ Complexity } & 0.0486 & 0.0475 & 0.0437 \\
\hline \multicolumn{2}{|c|}{$\begin{array}{l}\text { Airframe- } \\
\text { length/wing } \\
\text { span }\end{array}$} & 2.4873 & 2.8643 & 1.6978 \\
\hline
\end{tabular}

Table2. Experimental data

\begin{tabular}{|l|l|l|l|l|}
\hline \multicolumn{2}{|l|}{} & SU-27 & F-15 & Mig-29 \\
\hline $\begin{array}{l}\text { center } \\
\text { point }\end{array}$ & $\mathrm{x}$ & 258.133 & 248.620 & 200.904 \\
\cline { 2 - 5 } & $\mathrm{y}$ & 148.006 & 174.770 & 167.698 \\
\hline Direction & -0.076 & -0.437 & 0.087 \\
\hline Perimeter & 955 & 1145 & 1121 \\
\hline Area & 17699 & 21428 & 25885 \\
\hline Macro axis & 361 & 350 & 436 \\
\hline Minor axis & 134 & 227 & 209 \\
\hline $\begin{array}{l}\text { Compact- } \\
\text { ness }\end{array}$ & 0.3725 & 0.2806 & 0.2972 \\
\hline Complexity & 0.0540 & 0.0534 & 0.0444 \\
\hline $\begin{array}{l}\text { Airframe- } \\
\text { length/wing } \\
\text { span }\end{array}$ & 2.6940 & 1.5419 & 2.1373 \\
\hline
\end{tabular}

\subsection{Validating by SVM}

There are three steps to validate the effectiveness of features vector by SVM:

(1) modeling phase, (2)learning phase, (3) predicting phase.

Commonly, the model of SVM to recognize the target image is illustrated by Fig. 2 . 


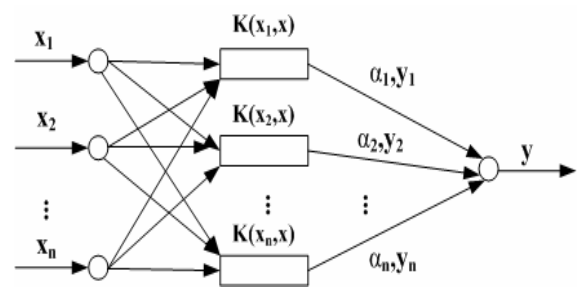

Fig.2 the model of SVM

In Fig.2, $K\left(x_{i}, x_{j}\right)$ is called by

Kernel Function. At present, most SVM select RBF as Kernel Function. RBF is a function of exponent:

$$
K\left(x \cdot x_{i}\right)=\exp \left(\frac{-\left\|x-x_{i}\right\|}{2 \sigma^{2}}\right)
$$

In this paper, we choose the software package LIBSVM to complete train and prediction, taking three-quarter data of total sample space to compose the training sample set, aim to train, taking a quarter data to compose the test sample set, aim to predict. The parameter configuration of LIBSVM and experiment result is reported by table 3 .

Table3. Result by LIBSVM

\begin{tabular}{|c|c|c|c|c|}
\hline 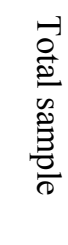 & 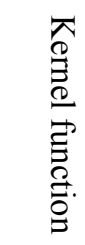 & 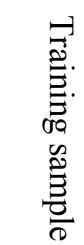 & 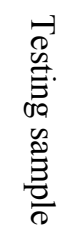 & 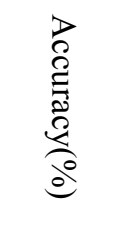 \\
\hline 45 & $\mathrm{RBF}$ & 36 & 9 & 85.7143 \\
\hline 80 & RBF & 60 & 20 & 86.0125 \\
\hline 125 & RBF & 94 & 31 & 87.2617 \\
\hline
\end{tabular}

\section{Conclusion}

See from Table 3, the accurateness is tend to increase by enhance the number to total sample. In case that the condition of experiment is allowable, we can further enhance the accurateness of prediction. But the rate of recognition $80 \%$ can illu- minate that these features vector is effective.

\section{References}

[1] Dudani S.A.Breeding K J.and MeGhee R B.Aircraft identification by moment invariant.IEEE trans.Compute, 1997,C-26(1):39 46

[2] Somaie A.A,Badr.A.and Salah T.Aircraft image recognition using back-propagation.CIE International Conference of Radar Proceedings, 2001:498 501

[3] Shen-Chi Tien,Tsorng-Lin Chia and Yibin Lu.Using cross-ratios to model curve data for aircraft recognition.Pattern Recognition Letters 2003,24:2047 2060

[4] Mokhtarian F.Silhouette-based occluded object recognition through curvature scale space.Machine Vision and Applications, 1997,13(10):87 97

[5] HAIT,SAWHNEYHS,KUMAR R. Object Tracking with Bayesian Estimation of Dynamic Layer Representations[J]. IEEE Transactions on Pattern Analysis and Machine Intelligence, 2002,24(1):75 $\sim 89$

[6] Saykol E,Gulesir G,Gudukbay U,et,al.A Kinematics-Based Method for Polygon Approximation[A].Advances in Information System(ADVIS'2002) [C].[s.1.]: [s.n.], 2002:186 194

[7] Marr,D.,Hildreth,E.Theory of Edge Detection.Proc.R.Soc.Lond,1980,B207:187 $\sim 217$

[8] Jan Flusser. Affine invariant of convex polygos[J].IEEE Trans. Image Processing,2002,11(9):1117 1118

[9] Scholkopf B.Statistical Learning and Kernel Methods[R].Microsoft Research Limited.http://research.microsoft.com/ bs c, 2000 